\title{
Vorbeugen ist (nicht immer) besser als heilen
}

\author{
Hans Stalder \\ Prof. Dr. med., Redaktionsmitglied
}

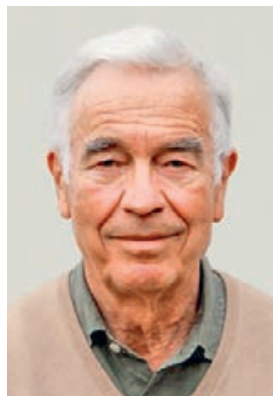

Die Literatur findet sich unter www.saez.ch

$\rightarrow$ Aktuelle Ausgabe oder $\rightarrow$ Archiv $\rightarrow 2019 \rightarrow 15$.

hans.stalder[at]saez.ch
Medizinische Vorsorge ist zweifellos etwas Gutes, und wer wäre für die Durchführung geeigneter als Ärztinnen und Ärzte? Aber seit dem eklatanten Misserfolg der Hormontherapie bei Frauen in der Menopause ist Vorsicht angesagt. Grundsätzlich folgte die Idee einer gewissen Logik: Nach der Menopause nehmen die weiblichen Hormone ab und die Frauen altern. Folglich müssen die Hormone ersetzt werden, um den Alterungsprozess zu stoppen. Von der Industrie unterstützte und von einigen Spezialisten propagierte Kohortenstudien schienen dies zu beweisen. Erst randomisierte und prospektive Studien, die Ko-Faktoren wie sozio-ökonomische Unterschiede ausschlossen, konnten zeigen, dass Hormone in der Tat schädlich sind. Diese Feststellung veranlasste David Sackett zu einem Leitartikel mit dem Titel The arrogance of preventive medicine, in dem er sich u.a. so äussert: «Without evidence from positive randomized trials (and, better still, systematic reviews of randomized trials) we cannot justify soliciting the well to accept any personal health intervention.» (Sackett DL. The arrogance of preventive medicine. CMAJ. 2002;167:363-4.) Inzwischen wurden weitere primäre Präventivmassnahmen aufgegeben: kein Aspirin, keine Omega-3Fettsäuren, kein Vitamin A oder E mehr für jedermann und nicht einmal mehr Vitamin D und Kalzium für ältere Menschen ...

Auch Screening-Massnahmen werden inzwischen in Frage gestellt. Die Bestimmung des PSA wird nicht mehr empfohlen. Die Mammographie ist umstritten. Das Screening auf Hypothyreose oder die routinemässige Prüfung auf Karotisgeräusche ist nicht mehr angezeigt, auch nicht das generelle Osteoporosescreening. Warum hatten wir alle diese Präventivmassnahmen ergriffen? Weil Prävention bestechend klingt, wenn die Ergebnisse als relative Reduktion eines pathologischen Prozesses dargestellt werden. So beispielsweise im Fall der Osteoporose, wo die medikamentöse Intervention das Risiko einer Hüftgelenksfraktur um mehr als 30\% gesenkt hat. Beeindruckend? In absoluten Zahlen weniger. Die Inzidenz ging nach fünf Jahren von 3,5 auf 2,6\% zurück [1]. Die Anzahl der notwendigen Behandlungen (NNT), um eine Fraktur zu vermeiden, beläuft sich somit auf 111. Aber für den Hausarzt (oder für das Gesundheitswesen) zählen nicht nur die NNT, sondern vor allem auch die
NNS (number necessary to screen) [2]. Bei einer Osteoporoseinzidenz bei Frauen von 15\% ist die NNS (um einen Fall zu erkennen) 6,6. Bei einer NNT von 111 müssen sich somit 733 Frauen einem Screening unterziehen, wovon $111 \mathrm{zu}$ behandeln sind, um eine Fraktur zu vermeiden ... Daher ist heute nicht nur die Früherkennung von Osteoporose [3], sondern auch die von Diabetes bei einer Population ohne Risiko umstritten [4]. Übrigens: Wussten Sie, dass die Prävention eines Glaukoms durch Früherkennung [5], die Prävention von Hautkrebs durch Sonnencreme [6] und die Prävention von Hauttumoren durch Früherkennung [7] nie durch ernstzunehmende Studien nachgewiesen werden konnten?

Ein weiteres Problem besteht darin, dass die Früherkennung und die daraus folgende Präventivbehandlung häufig nur die Inzidenz der getesteten Erkrankung verringern, nicht aber die Mortalität insgesamt und häufig auch nicht die Morbidität. Dies gilt für die Früherkennung von Brustkrebs durch die Mammographie oder das Screening auf Aortenaneurysma. Selbst die Endoskopie oder die Untersuchung des Stuhls auf Blut für das Screening auf Darmkrebs führt nicht zu einer Verringerung der Totalmortalität. Schwierig zu sagen, was Sterben leichter macht: Krebs oder (wahlweise) Herzinfarkt, Schlaganfall oder Demenz ...

Soll in der Arztpraxis also auf Prävention verzichtet werden? Keinesfalls! Es gilt jedoch, mit Bedacht vorzugehen, wenn primäre Prävention (eigentliche eher eine Angelegenheit der Gesundheitspolitik) ohne Absicherung durch prospektive, randomisierte Studien für die ganze Bevölkerung eingesetzt werden soll. Dies gilt auch für das Screening. Diese Mahnung richtet sich vor allem auch an die Fachgesellschaften, damit sie nicht in den Verdacht geraten, solche Massnahmen aus finanziellen Gründen zu empfehlen, wie das bei der Industrie der Fall ist. Ein anderes Problem ist die Anwendung von sekundären Präventivmassnahmen bei Risikopatienten. Dies ist nicht einfach, da sie personalisiert angewandt werden müssen. Mit Hilfe von Genanalysen? Warum nicht, aber dann wäre durch prospektive, randomisierte Studien zu beweisen, dass solche Analysen der einfachen familiären und persönlichen Anamnese und einer guten klinischen Untersuchung überlegen sind.

Less is more - dies gilt auch für die Prävention! 